\title{
BOUNDARY CONTROLLABILITY OF NONLINEAR STOCHASTIC FRACTIONAL SYSTEMS IN HILBERT SPACES
}

\author{
RAJENDRAN MABEL LIZZY ${ }^{a, *}$, KRISHNAN BALACHANDRAN $^{a}$ \\ ${ }^{a}$ Department of Mathematics \\ Bharathiar University, Coimbatore 641 046, India \\ e-mail: \{mabel.math.bu, kb.math. .bu\}@gmail.com
}

\begin{abstract}
Sufficient conditions for the controllability of nonlinear stochastic fractional boundary control systems are established. The equivalent integral equations are derived for both linear and nonlinear systems, and the control function is given in terms of the pseudoinverse operator. The Banach contraction mapping theorem is used to obtain the result. A controllability result for nonlinear stochastic fractional integrodifferential systems is also attained. Examples are included to illustrate the theory.
\end{abstract}

Keywords: boundary controllability, stochastic fractional systems, pseudoinverse, integrodifferential systems.

\section{Introduction}

Although there is extensive literature on controllability of spatiotemporal systems with control applied over the whole spatial domain, relatively little has been published on boundary controllability. In most applications however, control may be practically applied only on the boundary of the domain. An application where we encounter systems in which it is possible to exert control only on the boundary is a marine riser system where the controller is used to stabilize the riser, at the top end of the riser, as presented by Lasiecka and Triggiani (1991). Picard et al. (2012) studied the boundary control problem in viscoelasticity, and Hansen (1994) showed that the temperature flux control at one end of the one-dimensional thermoelastic rod is sufficient to obtain controllability.

There are many more applications for boundary control systems (Lagnese, 1977; Lions and Magenes, 1972; Washburn, 1979). Fattorini (1968) studied boundary control systems with first and second order time derivatives by converting the system to an equivalent one with control on the domain (distributed control) assuming that the control functions are differentiable, which is indeed a severe requirement and is highly impractical. This assumption is excluded by constructing a mild solution to the boundary control problem which can be seen in the works of Balakrishnan (1977) and Barbu (1980). Curtain and Zwart (1995) eliminated this

*Corresponding author assumption by reformulating the system in an extended state space. Boundary controllability of nonlinear systems with nonlocal conditions and integrodifferential systems was discussed by Han and Park (1999) as well as Balachandran and Anandhi (2001), respectively, using the construction of a pseudoinverse operator as in the work of Quinn and Carmichael (1985). Constrained controllability of systems in which controls are restricted to take values only in a prescribed set are studied by Klamka (1997; 2000), while boundary controllability of differential inclusions by Li and Liu (2008).

In the past few decades differential equations with fractional derivatives have proven to be a valuable tool to model many physical phenomena which even cover Abel's classical integral equations. Other well known fractional models include an analysis of feedback amplifiers, fractional order Chua-Hartley systems, fractional order models of neurons and many more (Kilbas et al., 2006; Podlubny, 1999). The time fractional diffusion-wave model as well as parabolic and elliptic equations with fractional diffusion were studied by Gal and Warma (2016), Mainardi et al. (2010) as well as Oprzędkiewicz et al. (2016).

Many authors have studied the controllability of fractional dynamical sytems (Balachandran and Divya, 2014; Balachandran and Kokila, 2012). Mathematical modeling is not complete until it incorporates the fluctuations in nature as noise terms yielding stochastic differential equations. The importance of the analysis 
of the qualitative behavior of systems with bounded operators is explained by Triggiani (1975). These factors motivate the study of stochastic fractional systems and investigation of their qualitative behavior. One type of qualitative behavior, namely, controllability for stochastic fractional systems with a bounded operator, is characterized by Mabel Lizzy et al. (2017) using the solution representation obtained by Balachandran et al. (2016). In this paper, we study the controllability of linear, nonlinear and integrodifferential nonlinear stochastic fractional systems with controls on the boundary. The systems are shown to be controllable by first deriving an equivalent integral equation and using the construction of a pseudoinverse operator to define the control function, and finally applying the Banach contraction mapping theorem in an appropriate space to obtain the desired result.

\section{Preliminaries}

Let $X, U, E$ and $H$ be separable Hilbert spaces. For convenience, we will use the same notation $\|\cdot\|$ to represent their norms. $\mathbb{L}(X, U)$ is the space of all bounded linear operators from $X$ to $U$ and $J$ denotes the interval $[0, T], T<+\infty$.

We assume that a filtered probability space $\left(\Omega, \mathcal{F},\left\{\mathcal{F}_{t}\right\}_{t \geq 0}, \mathbf{P}\right)$ is given with a probability measure $\mathbf{P}$ on $\Omega$, satisfying the "usual hypothesis":

(i) $\mathcal{F}_{0}$ contains all $F \in \mathcal{F}$ such that $\mathbf{P}(F)=0$,

(ii) $\mathcal{F}_{t}=\mathcal{F}_{t^{+}}, \forall t \in J$, where $\mathcal{F}_{t^{+}}$is the intersection of all $\mathcal{F}_{s}$ with $s>t$, i.e., the filtration is right continuous.

$W(t)$ is an $H$-valued Wiener process on the filtered probability space with covariance $t Q$, where $Q$ is a symmetric, nonnegative and bounded linear operator. Let us consider the following space settings:

- $Y:=\mathbb{L}_{2}\left(\Omega, \mathcal{F}_{T}, X\right)$ is a closed subspace of $\mathbb{L}_{2}(\Omega, X)$ consisting of all $\mathcal{F}_{T}$-measurable square integrable random variables with values in $X$.

- $\mathcal{H}_{2}$ is a closed subspace of $C\left(J, \mathbb{L}_{2}(\Omega, X)\right)$ consisting of all $\mathcal{F}_{t}$-measurable processes with values in $X$, identifying processes which are a modification of each other and endowed with the norm

$$
\|\phi\|_{\mathcal{H}_{2}}^{2}=\sup _{t \in J} \mathbf{E}\|\phi(t)\|^{2}
$$

where $\mathbf{E}$ denotes expectation with respect to $\mathbf{P}$.

- $U_{a d}:=\mathbb{L}_{2}^{\mathcal{F}}(J, U)$, which is a Hilbert space of all square integrable and $\mathcal{F}_{t}$-measurable processes with values in $U$.
- $\mathcal{H}_{2}^{0}:=\mathbb{L}_{2}\left(\Omega, \mathcal{F}_{0}, X\right)$, which is a Hilbert space of all $\mathcal{F}_{0}$-measurable square integrable random variables with values in $X$.

- Let $\left\{e_{n}\right\}_{n=1}^{\infty} \subset Q^{1 / 2} H$ be a complete orthonormal basis. We denote by $\mathbb{L}_{2}^{0}=\mathbb{L}_{2}\left(Q^{1 / 2} H, X\right)$ the space of Hilbert-Schmidt operators from $Q^{1 / 2} H$ to $X$ with the norm given by

$$
\|\sigma\|_{\mathbb{L}_{2}^{0}}=\left(\sum_{k=0}^{\infty}\left\|\sigma e_{n}\right\|_{X}^{2}\right)^{1 / 2} .
$$

Let us recall some basic definitions from fractional calculus. Let $\alpha, \beta>0$, with $n-1<\alpha<n, n-1<$ $\beta<n$ and $n \in \mathbb{N}$ and $f \in L_{1}([0, \infty))$.

Definition 1. (Kilbas et al., 2006) By the RiemannLiouville fractional integral of a function $f$ we mean

$$
I^{\alpha} f(t)=\frac{1}{\Gamma(\alpha)} \int_{0}^{t}(t-s)^{\alpha-1} f(s) \mathrm{d} s,
$$

and the Caputo derivative of $f$ is ${ }^{C} D^{\alpha} f=I^{n-\alpha} D^{n} f$, that is,

$$
{ }^{C} D^{\alpha} f(t)=\frac{1}{\Gamma(n-\alpha)} \int_{0}^{t}(t-s)^{n-\alpha-1} f^{(n)}(s) \mathrm{d} s,
$$

where the function $f(t)$ has absolutely continuous derivatives up to order $n-1$ and $\Gamma(\cdot)$ represents the gamma function.

Definition 2. (Kilbas et al., 2006) Let $A$ be a bounded linear operator. The two parameter Mittag-Leffler operator function is given by

$$
E_{\alpha, \beta}(A)=\sum_{k=0}^{\infty} \frac{A^{k}}{\Gamma(\alpha k+\beta)} .
$$

In particular, taking $\beta=1$ we get the one parameter Mittag-Leffler operator function,

$$
E_{\alpha, 1}(A)=E_{\alpha}(A)=\sum_{k=0}^{\infty} \frac{A^{k}}{\Gamma(\alpha k+1)} .
$$
form

Consider the linear boundary control problem of the

$$
\begin{aligned}
{ }^{C} D^{\alpha} x(t) & =A_{1} x(t)+f(t)+\sigma(t) \frac{\mathrm{d} W(t)}{\mathrm{d} t}, \quad t \in J, \\
\tau x(t) & =B_{1} u(t), \\
x(0) & =x_{0} \in \mathcal{H}_{2}^{0},
\end{aligned}
$$

where $0<\alpha \leq 1, A_{1}$ and $B_{1}$ are bounded linear operators and $\tau$ is a linear operator with $D\left(A_{1}\right) \subset$ $X, D(\tau) \subset X, R(\tau) \subset E, W(t)$ is an infinite dimensional Wiener process. $f: J \rightarrow X$ is a continuous 
function and $\sigma(t)$ is a Hilbert-Schmidt operator for all $t \in J$. Let $A: X \rightarrow X$ be a linear operator defined by

$$
\begin{aligned}
D(A) & =\left\{x \in D\left(A_{1}\right): \tau x=0\right\}, \\
A x & =A_{1} x, \quad \forall x \in D(A) .
\end{aligned}
$$

Our basic assumptions are formulated as the following hypotheses.

(H1) $D\left(A_{1}\right) \subseteq D(\tau)$.

(H2) The bounded linear operator $A$ satisfies

$$
\|A\|^{2}<\frac{(2 \alpha-1)(\Gamma(\alpha))^{2}}{T^{2 \alpha}}
$$

(H3) There exists a linear operator $B: U \rightarrow X$ such that for all $u \in U$ we have $B u \in D\left(A_{1}\right), \tau(B u)=B_{1} u$ and $\|B u\|^{2} \leq C\left\|B_{1} u\right\|^{2}$, where $C$ is a constant.

(H4) For all $t \in J$ and $u \in U$ we have $E_{\alpha, \alpha}\left(A t^{\alpha}\right) B u \in$ $D(A)$.

Let $x(t)$ be the solution of (1); then we can define a function $z(t)=x(t)-B u(t)$, and from the hypothesis (H3) we see that $z(t) \in D(A)$. Hence (1) is written in terms of $A$ and $B$ as

$$
\begin{aligned}
{ }^{C} D^{\alpha} z(t)= & A z(t)+A_{1} B u(t)-B^{C} D^{\alpha} u(t)+f(t) \\
& +\sigma(t) \frac{\mathrm{d} W(t)}{\mathrm{d} t}, \quad t \in J \\
z(0)= & x_{0}-B u(0) .
\end{aligned}
$$

Applying $I^{\alpha}$ to both the sides of (2), we have

$$
\begin{aligned}
z(t)= & x_{0}+I^{\alpha} A z(t)+I^{\alpha} A_{1} B u(t)-B u(t)+I^{\alpha} f(t) \\
& +I^{\alpha} \sigma(t) \frac{\mathrm{d} W(t)}{\mathrm{d} t} \\
= & \left(I-I^{\alpha} A\right)^{-1}\left(x(0)+I^{\alpha} A_{1} B u(t)-B u(t)\right. \\
& \left.+I^{\alpha} f(t)+I^{\alpha} \sigma(t) \frac{\mathrm{d} W(t)}{\mathrm{d} t}\right),
\end{aligned}
$$

where $I$ denotes the identity operator on $X$. Using (H2) we see that

$$
\begin{aligned}
& \left\|\left(I^{\alpha} A\right) x\right\|_{\mathcal{H}_{2}} \\
& \quad \leq \frac{T}{(\Gamma(\alpha))^{2}} \sup _{t \in J} \int_{0}^{t}(t-s)^{2 \alpha-2} \mathbf{E}\|A x(s)\|_{X}^{2} \mathrm{~d} s \\
& \quad \leq \frac{T^{2 \alpha}}{(2 \alpha-1)(\Gamma(\alpha))^{2}} \sup _{t \in J} \mathbf{E}\|A x\|_{X}^{2}<\|x\|_{\mathcal{H}_{2}}
\end{aligned}
$$

Lemma 1. (Kreyszig, 1978) Assume that $A$ is a linear bounded operator defined on a Banach space and $\|A\|<$ 1. Then $(I-A)^{-1}$ is linear and bounded. Moreover,

$$
(I-A)^{-1}=\sum_{k=0}^{\infty} A^{k} .
$$

The convergence of the above series is in the operator norm, and $\left\|(I-A)^{-1}\right\| \leq(1-\|A\|)^{-1}$.

With Lemma 1 we obtain the solution of (1) in terms of the Mittag-Leffler operator function as in the work of Balachandran et al. (2016) as follows:

$$
\begin{aligned}
& x(t) \\
& =\sum_{k=0}^{\infty}\left(I^{\alpha} A\right)^{k}\left(x_{0}-B u(t)+I^{\alpha} A_{1} B u(t)\right. \\
& \left.+I^{\alpha} f(t)+I^{\alpha} \sigma(t) \frac{\mathrm{d} W(t)}{\mathrm{d} t}\right)+B u(t) \\
& =\sum_{k=0}^{\infty} I^{k \alpha} A^{k} x_{0}-\sum_{k=1}^{\infty} I^{k \alpha} A^{k} B u(t) \\
& +\sum_{k=0}^{\infty} I^{k \alpha+\alpha} A^{k}\left[A_{1} B u(t)+f(t)+\sigma(t) \frac{\mathrm{d} W(t)}{\mathrm{d} t}\right] \\
& =\sum_{k=0}^{\infty} I^{k \alpha} A^{k} x_{0}+\sum_{k=0}^{\infty} I^{k \alpha+\alpha} A^{k}[-A B u(t) \\
& \left.+A_{1} B u(t)+f(t)+\sigma(t) \frac{\mathrm{d} W(t)}{\mathrm{d} t}\right] \\
& =\sum_{k=0}^{\infty} \frac{A^{k} t^{\alpha k}}{\Gamma(k \alpha+1)} x_{0} \\
& -\int_{0}^{t} A(t-s)^{\alpha-1}\left(\sum_{k=0}^{\infty} \frac{A^{k}(t-s)^{\alpha k}}{\Gamma(k \alpha+\alpha)}\right) B u(s) \mathrm{d} s \\
& +\int_{0}^{t}(t-s)^{\alpha-1}\left(\sum_{k=0}^{\infty} \frac{A^{k}(t-s)^{\alpha k}}{\Gamma(k \alpha+\alpha)}\right) \\
& \times\left[A_{1} B u(s)+f(s)\right] \mathrm{d} s \\
& +\int_{0}^{t}(t-s)^{\alpha-1}\left(\sum_{k=0}^{\infty} \frac{A^{k}(t-s)^{\alpha k}}{\Gamma(k \alpha+\alpha)}\right) \sigma(s) \mathrm{d} W(s) .
\end{aligned}
$$

The hypothesis (H4) validates the appearance of the term $\left.A E_{\alpha, \alpha}\left(A(t-s)^{\alpha}\right)\right] B u(s)$, and the solution is obtained as

$$
\begin{aligned}
& x(t) \\
& =E_{\alpha}\left(A t^{\alpha}\right) x_{0} \\
& \quad+\int_{0}^{t}(t-s)^{\alpha-1}\left[E_{\alpha, \alpha}\left(A(t-s)^{\alpha}\right) A_{1}\right. \\
& \left.\quad-A E_{\alpha, \alpha}\left(A(t-s)^{\alpha}\right)\right] B u(s) \mathrm{d} s \\
& \quad+\int_{0}^{t}(t-s)^{\alpha-1} E_{\alpha, \alpha}\left(A(t-s)^{\alpha}\right) f(s) \mathrm{d} s
\end{aligned}
$$




$$
+\int_{0}^{t}(t-s)^{\alpha-1} E_{\alpha, \alpha}\left(A(t-s)^{\alpha}\right) \sigma(s) \mathrm{d} W(s) .
$$

Controllability for an infinite dimensional deterministic system in $X$ means that, for each pair of points $x_{0}, x_{1} \in$ $X$, there exists a control in the admissible control space such that it steers the solution of the system from the initial point $x_{0}$ to the final point $x_{1}$. The same definition of controllability can be extended to fractional systems (Balachandran et al., 2016), but for stochastic systems this does not make any sense and so a different notion of controllability is required. The existing literature suggests two different ways of extending the controllability concepts to stochastic systems, which are given as follows:

- the property of attaining all states in a suitable space of random variables, for example, the space of square integrable random variables;

- the property of attaining an arbitrarily small neighborhood of each point in the state space with a probability arbitrarily close to one, fortified with some uniformity.

In the former approach the state space consists of random variables whereas in the latter it includes only nonrandom values. These approaches were studied by Bashirov (2003) and Mahmudov (2001; 2003), respectively. We define the controllability of the stochastic fractional dynamical system similarly to the conventional controllability concept for stochastic systems in the work of Mahmudov (2001) as follows.

The set of all states attainable from $x_{0}$ in time $t>0$ is given by the set

$$
\mathcal{R}_{t}\left(x_{0}\right)=\left\{x(t): u \in U_{\mathrm{ad}}\right\},
$$

where $x(t)$ is given in (3).

Definition 3. The stochastic fractional dynamical system (1) is said to be completely controllable on the interval $J$ if for every $x_{1} \in Y$ there exists a control $u \in U_{\text {ad }}$ such that the solution $x(t)$ given in (3) satisfies $x(T)=x_{1}$. In other words,

$$
\mathcal{R}_{T}\left(x_{0}\right)=Y .
$$

Let us consider the bounded linear operator $L_{T}$ : $U_{\text {ad }} \rightarrow Y$ defined by

$$
\begin{aligned}
L_{T} u(t)= & \int_{0}^{T}\left[E_{\alpha, \alpha}\left(A(T-t)^{\alpha}\right) A_{1}\right. \\
& \left.-A E_{\alpha, \alpha}\left(A(T-t)^{\alpha}\right)\right] B u(t) \mathrm{d} t .
\end{aligned}
$$

Since the kernel of $L_{T}$, $\operatorname{ker} \mathrm{L}_{\mathrm{T}}$ is closed, the space $U^{0}=$ $U_{\mathrm{ad}} / \mathrm{kerL}_{\mathrm{T}}$ is a Hilbert space under the following norm:

$$
\|[u]\|_{U^{0}}:=\max _{u \in[u]}\|u\|_{U_{\mathrm{ad}}}=\max _{L_{T} \hat{u}=0}\|u+\hat{u}\|_{U_{\mathrm{ad}}},
$$

where $u \in[u]$. We define $\tilde{L}_{T}: U_{\text {ad }} / \operatorname{ker} \mathrm{L}_{\mathrm{T}} \rightarrow \mathrm{Y}$ as $\tilde{L}_{T}[u]:=L_{T} u, u \in[u]$. We can show that $\tilde{L}_{T}$ is a one-to-one mapping and we also see that it is continuous. Indeed,

$$
\begin{aligned}
\left\|\tilde{L}_{T}[u]\right\|_{Y} & =\left\|L_{T} u\right\|_{Y} \leq\left\|L_{T}\right\|\|u\|_{Y} \\
& \leq\left\|L_{T}\right\|\|[u]\|_{U^{0}}, \quad u \in[u] .
\end{aligned}
$$

By the bounded inverse theorem we now obtain that $\tilde{L}_{T}$ has a bounded inverse on $Y$, i.e., $L_{T} \tilde{L}_{T}=I$, and there exists a constant $K>0$ such that $\left\|\tilde{L}_{T}^{-1}\right\|^{2} \leq K$.

Theorem 1. If the hypotheses (H1)-(H4) are satisfied, then the linear system (1) is completely controllable.

Proof. Using (H1)-(H4), we obtain the solution of (1) as in (3). Let $x_{1}$ be an arbitrary point in $Y$. Since the linear operator $\tilde{L}_{T}$ is invertible, we define the control process as

$$
\begin{aligned}
& u(t) \\
& =(T-t)^{1-\alpha} \tilde{L}_{T}^{-1}\left(x_{1}-E_{\alpha}\left(A T^{\alpha}\right) x_{0}\right. \\
& \quad-\int_{0}^{T}(T-s)^{\alpha-1} E_{\alpha, \alpha}\left(A(T-s)^{\alpha}\right) f(s) \mathrm{d} s \\
& \left.\quad-\int_{0}^{T}(T-s)^{\alpha-1} E_{\alpha, \alpha}\left(A(T-s)^{\alpha}\right) \sigma(s) \mathrm{d} W(s)\right) .
\end{aligned}
$$

Substituting (4) in (3) we get

$$
\begin{aligned}
x(t) & \\
= & E_{\alpha}\left(A T^{\alpha}\right) x_{0}+\int_{0}^{t}(t-s)^{\alpha-1}\left[E_{\alpha, \alpha}\left(A(t-s)^{\alpha}\right) A_{1}\right. \\
& \left.-A E_{\alpha, \alpha}\left(A(t-s)^{\alpha}\right)\right] B(T-s)^{1-\alpha} \\
& \tilde{L}_{T}^{-1}\left(x_{1}-E_{\alpha}\left(A T^{\alpha}\right) x_{0}\right. \\
& -\int_{0}^{T}(T-r)^{\alpha-1} E_{\alpha, \alpha}\left(A(T-r)^{\alpha}\right) f(r) \mathrm{d} r \\
& \left.-\int_{0}^{T}(T-r)^{\alpha-1} E_{\alpha, \alpha}\left(A(T-r)^{\alpha}\right) \sigma(r) \mathrm{d} W(r)\right) \mathrm{d} s \\
& +\int_{0}^{t}(t-s)^{\alpha-1} E_{\alpha, \alpha}\left(A(t-s)^{\alpha}\right) f(s) \mathrm{d} s \\
& +\int_{0}^{t}(t-s)^{\alpha-1} E_{\alpha, \alpha}\left(A(t-s)^{\alpha}\right) \sigma(s) \mathrm{d} W(s) .
\end{aligned}
$$

Evaluating $x(t)$ given in the above equation at $t=T$ we obtain

$$
\begin{aligned}
& x(T) \\
& =E_{\alpha}\left(A T^{\alpha}\right) x_{0}+L_{T} \tilde{L}_{T}^{-1}\left(x_{1}-E_{\alpha}\left(A T^{\alpha}\right) x_{0}\right. \\
& \quad-\int_{0}^{T}(T-r)^{\alpha-1} E_{\alpha, \alpha}\left(A(T-r)^{\alpha}\right) f(r) \mathrm{d} r
\end{aligned}
$$




$$
\begin{aligned}
& \left.-\int_{0}^{T}(T-r)^{\alpha-1} E_{\alpha, \alpha}\left(A(T-r)^{\alpha}\right) \sigma(r) \mathrm{d} W(r)\right) \mathrm{d} s \\
& +\int_{0}^{T}(T-s)^{\alpha-1} E_{\alpha, \alpha}\left(A(T-s)^{\alpha}\right) f(s) \mathrm{d} s \\
& +\int_{0}^{T}(T-s)^{\alpha-1} E_{\alpha, \alpha}\left(A(T-s)^{\alpha}\right) \sigma(s) \mathrm{d} W(s)=x_{1} .
\end{aligned}
$$

Since $x_{1}$ is an arbitrary point in $Y$, we infer from the above that $u(t)$ defined in (4) steers the system to all points in $Y$. This implies $\mathcal{R}_{T}\left(x_{0}\right)=Y$, and thus the proof is completed.

\section{Nonlinear system}

Consider the nonlinear boundary control problem of the form

$$
\begin{aligned}
{ }^{C} D^{\alpha} x(t)= & A_{1} x(t)+f(t, x(t)) \\
& +\sigma(t, x(t)) \frac{\mathrm{d} W(t)}{\mathrm{d} t}, \quad t \in J, \\
\tau x(t)= & B_{1} u(t), \\
x(0)= & x_{0} \in \mathcal{H}_{2}^{0},
\end{aligned}
$$

where $0 \leq \alpha<1, \alpha \neq 1 / 2, f: J \times X \rightarrow X$ and $\sigma: J \times X \rightarrow \mathbb{L}_{2}^{0}$ are continuous functions. We adopt the following notation:

$$
\begin{gathered}
M_{1}=\max _{t \in J}\left\|E_{\alpha}\left(A t^{\alpha}\right)\right\|_{X}^{2}, \\
M_{2}=\max _{0 \leq s \leq t \leq T}\left\|E_{\alpha, \alpha}\left(A(t-s)^{\alpha}\right)\right\|_{X}^{2}, \\
N_{1}=\max _{t \in J}\|f(t, 0)\|_{X}^{2}, \\
N_{2}=\max _{t \in J}\|\sigma(t, 0)\|_{\mathbb{L}_{2}^{0}}^{2},
\end{gathered}
$$

and further assume the following hypotheses.

(H5) Let

$$
\begin{aligned}
\rho_{1}=\frac{3 M_{2} T^{2 \alpha-1}}{2 \alpha-1}\left(T L_{1}+L_{2}\right)\left(1+4 M_{2} C K\right. \\
\left.\quad \times\left\|B_{1}\right\|^{2}\left(\left\|A_{1}\right\|^{2}+\frac{(2 \alpha-1)(\Gamma(\alpha))^{2}}{T^{2 \alpha}}\right)\right)
\end{aligned}
$$

be such that $0 \leq \rho_{1}<1$.

(H6) There exist constants $L_{i}>0, i=1,2$, such that

$$
\begin{gathered}
\left\|f\left(t, x_{1}\right)-f\left(t, x_{2}\right)\right\|_{X}^{2} \leq L_{1}\left\|x_{1}-x_{2}\right\|_{X}^{2}, \\
\left\|\sigma\left(t, x_{1}\right)-\sigma\left(t, x_{2}\right)\right\|_{\mathbb{L}_{2}^{0}}^{2} \leq L_{2}\left\|x_{1}-x_{2}\right\|_{X}^{2}
\end{gathered}
$$

for all $x_{1}, x_{2}, y_{1}, y_{2} \in X$.

Theorem 2. If the hypotheses of Theorem 1 along with (H5) and (H6) are satisfied, then the boundary control nonlinear stochastic fractional system (5) is completely controllable.
Proof. Let $x_{1}$ be an arbitrary random variable in $Y$. Using (H1)-(H4), we obtain the equivalent nonlinear integral equation for the system (5) in terms of the Mittag-Leffler operator function as

$$
\begin{aligned}
x(t) & \\
= & E_{\alpha}\left(A t^{\alpha}\right) x_{0}+\int_{0}^{t}(t-s)^{\alpha-1}\left[E_{\alpha, \alpha}\left(A(t-s)^{\alpha}\right) A_{1}\right. \\
& \left.-A E_{\alpha, \alpha}\left(A(t-s)^{\alpha}\right)\right] B u(s) \mathrm{d} s \\
& +\int_{0}^{t}(t-s)^{\alpha-1} E_{\alpha, \alpha}\left(A(t-s)^{\alpha}\right) f(s, x(s)) \mathrm{d} s \\
& +\int_{0}^{t}(t-s)^{\alpha-1} E_{\alpha, \alpha}\left(A(t-s)^{\alpha}\right) \sigma(s, x(s)) \mathrm{d} W(s) .
\end{aligned}
$$

We now define the operator $\Phi$ on $\mathcal{H}_{2}$ by $\Phi x(t)$ to be the right hand side of the above equation. The invertibility of $\tilde{L}_{T}$ allows us to choose the control process $u$ as

$$
\begin{aligned}
u(t)= & (T-t)^{1-\alpha} \tilde{L}_{T}^{-1}\left(x_{1}-E_{\alpha}\left(A T^{\alpha}\right) x_{0}\right. \\
& -\int_{0}^{T}(T-s)^{\alpha-1} E_{\alpha, \alpha}\left(A(T-s)^{\alpha}\right) f(s, x(s)) \mathrm{d} s \\
& -\int_{0}^{T}(T-s)^{\alpha-1} E_{\alpha, \alpha}\left(A(T-s)^{\alpha}\right) \\
& \sigma(s, x(s)) \mathrm{d} W(s)) .
\end{aligned}
$$

The control $u$ steers the nonlinear system from the initial state $x_{0}$ to $x_{1}$ at time $T$, provided we can obtain a fixed point of the nonlinear operator $\Phi$. First we show that $\Phi$ maps $\mathcal{H}_{2}$ into itself. From the assumption (H6) and the adopted notion, we have

$$
\begin{array}{rl}
\sup _{t \in J} & \mathbf{E}\|\Phi x(t)\|^{2} \\
\leq & 4 M_{1} \mathbf{E}\left\|x_{0}\right\|^{2}+32 M_{2} C K\left\|B_{1}\right\|^{2}\left(\left\|A_{1}\right\|^{2}\right. \\
& \left.+\frac{(2 \alpha-1)(\Gamma(\alpha))^{2}}{T^{2 \alpha}}\right)\left(\mathbf{E}\left\|x_{1}\right\|^{2}+M_{1} \mathbf{E}\left\|x_{0}\right\|^{2}\right. \\
& +\frac{T^{2 \alpha}}{2 \alpha-1} M_{2}\left(L_{1} \sup _{t \in J} \mathbf{E}\|x(t)\|^{2}+N_{1}\right) \\
& \left.+\frac{T^{2 \alpha-1}}{2 \alpha-1} M_{2}\left(L_{2} \sup _{t \in J} \mathbf{E}\|x(t)\|^{2}+N_{2}\right)\right) \\
& +4 \frac{T^{2 \alpha}}{2 \alpha-1} M_{2}\left(L_{1} \sup _{t \in J} \mathbf{E}\|x(t)\|^{2}+N_{1}\right) \\
& +4 \frac{T^{2 \alpha-1}}{2 \alpha-1} M_{2}\left(L_{2} \sup _{t \in J} \mathbf{E}\|x(t)\|^{2}+N_{2}\right) .
\end{array}
$$

From the above inequality it follows that, if $\alpha \neq 1 / 2$, there exists a constant $C_{1}>0$ such that

$\sup _{t \in J} \mathbf{E}\|\Phi x(t)\|^{2} \leq C_{1}\left(1+\sup _{t \in J} \mathbf{E}\|x(t)\|^{2}\right)$. 
Thus $\Phi$ maps $\mathcal{H}_{2}$ into itself. Now for $x_{1}, x_{2} \in \mathcal{H}_{2}$ we have, using (H6), the following inequality:

$$
\begin{aligned}
\sup _{t \in J} & \left.\mathbf{E} \| \Phi x_{1}(t)-\Phi x_{2}(t)\right) \|_{X}^{2} \\
= & \sup _{t \in J} \mathbf{E} \| \int_{0}^{t}(t-s)^{\alpha-1}\left[E_{\alpha, \alpha}\left(A(t-s)^{\alpha}\right) A_{1}\right. \\
& \left.-A E_{\alpha, \alpha}\left(A(t-s)^{\alpha}\right)\right] B(T-s)^{1-\alpha} \tilde{L}_{T}^{-1} \\
& {\left[\int_{0}^{T}(T-\theta)^{\alpha-1} E_{\alpha, \alpha}\left(A(T-\theta)^{\alpha}\right)\right.} \\
& \left(\sigma\left(\theta, x_{1}(\theta)\right)-\sigma\left(\theta, x_{2}(\theta)\right)\right) \mathrm{d} W(\theta) \\
& +\int_{0}^{T}(T-\theta)^{\alpha-1} E_{\alpha, \alpha}\left(A(T-\theta)^{\alpha}\right) \\
& \left.\left(f\left(\theta, x_{1}(\theta)\right)-f\left(\theta, x_{2}(\theta)\right)\right) \mathrm{d} \theta\right] \mathrm{d} s \\
& +\int_{0}^{t}(t-s)^{\alpha-1} E_{\alpha, \alpha}\left(A(t-s)^{\alpha}\right) \\
& \left(f\left(s, x_{1}(s)\right)-f\left(s, x_{2}(s)\right)\right) \mathrm{d} s \\
& +\int_{0}^{t}(t-s)^{\alpha-1} E_{\alpha, \alpha}\left(A(T-s)^{\alpha}\right) \\
\leq & {\left[\sigma\left(s, x_{1}(s)\right)-\sigma\left(s, x_{2}(s)\right)\right] \mathrm{d} W(s) \|^{2} } \\
\leq & \frac{3 M_{2} T^{2 \alpha-1}\left(T L_{1}+L_{2}\right)}{2 \alpha-1} \sup _{t \in J} \mathbf{E}\left\|x_{1}(t)-x_{2}(t)\right\|_{X}^{2} \\
& \times\left(1+4 x_{2} C K\left\|B_{1}\right\|^{2}(t)-x_{2}(t) \|_{X}^{2} \cdot\right. \\
& \left.\times\left(\left\|A_{1}\right\|^{2}+\frac{(2 \alpha-1)(\Gamma(\alpha))^{2}}{T^{2 \alpha}}\right)\right) \\
& (f \alpha-1
\end{aligned}
$$

Using (H5), we conclude that $\Phi$ is a contraction mapping and hence there exists a unique fixed point $x \in \mathcal{H}_{2}$ for $\Phi$. Therefore the system (5) is completely controllable.

\section{Nonlinear time-varying system}

Consider the time-varying nonlinear boundary control system

$$
\begin{aligned}
{ }^{C} D^{\alpha} x(t)= & A_{1}(t) x(t)+f(t, x(t)) \\
& +\sigma(t, x(t)) \frac{\mathrm{d} W(t)}{\mathrm{d} t}, \quad t \in J, \\
\tau x(t)= & B_{1} u(t), \\
x(0)= & x_{0} \in \mathcal{H}_{2}^{0},
\end{aligned}
$$

where $A_{1}(t)$ is a bounded linear operator for all $t \in J$ with its domain independent of $t$ and denoted by $D\left(A_{1}\right) \subset X$. For all $t \in J$, let $A(t): X \rightarrow X$ be the linear operator defined by

$$
\begin{aligned}
D(A(t)) & =D(A)=\left\{x \in D\left(A_{1}\right): \tau x=0\right\}, \quad \forall t \in J, \\
A(t) x & =A_{1}(t) x, \quad \forall x \in D(A) .
\end{aligned}
$$

Since the operators $A(t)$ and $I^{\alpha}$ do not commute $\left(I^{\alpha} A\right)^{k} \neq I^{\alpha k} A^{k}$. The solution cannot be obtained in terms of the Mittag-Leffler operator function as in the previous section, and so we adopt a different approach to obtain the equivalent integral equation for the nonautonomous boundary control problem (6). Let $x(t)$ be the solution of (6); then we can define a function $z(t)=x(t)-B u(t)$, and from the hypothesis (H3) we see that $z(t) \in D(A)$. Hence (5) is written in terms of $A(t)$ and $B$ as

$$
\begin{aligned}
{ }^{C} D^{\alpha} z(t)= & A(t) z(t)+A_{1}(t) B u(t) \\
& -B^{C} D^{\alpha} u(t)+f(t, x(t)) \\
& +\sigma(t, x(t)) \frac{\mathrm{d} W(t)}{\mathrm{d} t}, \quad t \in J, \\
z(0)= & x_{0}-B u(0) .
\end{aligned}
$$

Applying $I^{\alpha}$ to both the sides of (7), we have the following integral equation equivalent to 6

$$
\begin{aligned}
x(t)= & x_{0}+I^{\alpha} A(t) x(t)-I^{\alpha} A(t) B u(t) \\
& +I^{\alpha} A_{1}(t) B u(t)+I^{\alpha} f(t, x(t)) \\
& +I^{\alpha} \sigma(t, x(t)) \frac{\mathrm{d} W(t)}{\mathrm{d} t} \\
= & x_{0}+\frac{1}{\Gamma(\alpha)} \int_{0}^{t}(t-s)^{\alpha-1} A(s) x(s) \mathrm{d} s \\
& +\frac{1}{\Gamma(\alpha)} \int_{0}^{t}(t-s)^{\alpha-1}\left[A_{1}(s)-A(s)\right] B u(s) \mathrm{d} s \\
& +\frac{1}{\Gamma(\alpha)} \int_{0}^{t}(t-s)^{\alpha-1} f(s, x(s)) \mathrm{d} s \\
& +\frac{1}{\Gamma(\alpha)} \int_{0}^{t}(t-s)^{\alpha-1} \sigma(s, x(s)) \mathrm{d} W(s) .
\end{aligned}
$$

The linear operator $L_{T}: U_{\mathrm{ad}} \rightarrow Y$ defined by

$$
L_{T} u=\frac{1}{\Gamma(\alpha)} \int_{0}^{T}(T-s)^{\alpha-1}\left[A_{1}(s)-A(s)\right] B u(s) \mathrm{d} s
$$

induces an invertible operator $\tilde{L}_{T}$ defined on $U_{\text {ad }} / \operatorname{ker} L_{T}$ and the inverse is bounded by a constant, i.e., $\left\|\tilde{L}_{T}^{-1}\right\|^{2} \leq$ $K$, for some $K>0$ as proved in the previous section. The following hypothesis is made.

(H7) Let

$$
\begin{aligned}
\rho_{2}= & \frac{4 T^{2 \alpha-1}}{(2 \alpha-1)(\Gamma(\alpha))^{2}}\left(\sup _{t \in J}\|A(t)\|^{2}+T L_{1}+L_{2}\right) \\
& \left(1+6 K \sup _{t \in J}\left\|A_{1}(t)\right\|^{2}\|B\|^{2}\right)
\end{aligned}
$$

be such that $0 \leq \rho_{2}<1$.

Theorem 3. If the hypotheses (H1), (H3), (H6) and (H7) are satisfied, then the boundary control nonlinear stochastic fractional system (6) is completely controllable. 
Proof. Let $x_{1}$ be an arbitrary random variable in $Y$. Using (H1) and (H3), we have obtained the equivalent integral equation for the system in the form given by (8). Define the operator $\Phi$ on $\mathcal{H}_{2}$ by

$\Phi x(t)$

$$
\begin{aligned}
= & x_{0}+\frac{1}{\Gamma(\alpha)} \int_{0}^{t}(t-s)^{\alpha-1} A(s) x(s) \mathrm{d} s \\
& +\frac{1}{\Gamma(\alpha)} \int_{0}^{t}(t-s)^{\alpha-1}\left[A_{1}(s)-A(s)\right] B u(s) \mathrm{d} s \\
& +\frac{1}{\Gamma(\alpha)} \int_{0}^{t}(t-s)^{\alpha-1} f(s, x(s)) \mathrm{d} s \\
& +\frac{1}{\Gamma(\alpha)} \int_{0}^{t}(t-s)^{\alpha-1} \sigma(s, x(s)) \mathrm{d} W(s) .
\end{aligned}
$$

The invertibility of $\tilde{L}_{T}$ allows us to choose the control process $u$ as

$$
\begin{aligned}
u(t)= & (T-t)^{1-\alpha} \tilde{L}_{T}^{-1}\left(x_{1}-x_{0}\right. \\
& -\frac{1}{\Gamma(\alpha)} \int_{0}^{T}(T-s)^{\alpha-1} A(s) x(s) \mathrm{d} s \\
& -\frac{1}{\Gamma(\alpha)} \int_{0}^{T}(T-s)^{\alpha-1} f(s, x(s)) \mathrm{d} s \\
& \left.-\frac{1}{\Gamma(\alpha)} \int_{0}^{T}(T-s)^{\alpha-1} \sigma(s, x(s)) \mathrm{d} W(s)\right) .
\end{aligned}
$$

The control $u$ steers the nonlinear system from the initial state $x_{0}$ to $x_{1}$ at time $T$, provided we can obtain a fixed point of the nonlinear operator $\Phi$. First we show that $\Phi$ maps $\mathcal{H}_{2}$ into itself. From the assumptions, we have

$$
\begin{aligned}
& \sup _{t \in J} \mathbf{E}\|\Phi x(t)\|^{2} \\
& \leq 50 C K \sup _{t \in J}\left\|A_{1}(t)\right\|^{2}\left\|B_{1}\right\|^{2} \mathbf{E}\left\|x_{1}\right\|^{2} \\
& \quad+\left(1+10 C K \sup _{t \in J}\left\|A_{1}(t)\right\|^{2}\left\|B_{1}\right\|^{2}\right) \\
& \quad \times\left(5 \mathbf{E}\left\|x_{0}\right\|^{2}+\frac{5 T^{2 \alpha}}{(\Gamma(\alpha))^{2}(2 \alpha-1)}\left(L_{1} \sup _{t \in J} \mathbf{E}\|x(t)\|^{2}\right.\right. \\
& \left.\left.\quad+N_{1}+T L_{2} \sup _{t \in J} \mathbf{E}\|x(t)\|^{2}+T N_{2}\right)\right) .
\end{aligned}
$$

From the above inequality it follows that, if $\alpha \neq 1 / 2$, there exists a constant $C_{2}>0$ such that

$$
\sup _{t \in J} \mathbf{E}\|\Phi x(t)\|^{2} \leq C_{2}\left(1+\sup _{t \in J} \mathbf{E}\|x(t)\|^{2}\right) .
$$

Thus $\Phi$ maps $\mathcal{H}_{2}$ into itself. Now, for $x_{1}, x_{2} \in \mathcal{H}_{2}$, using the Lipschitz conditions on the nonlinear functions in (H6), we have

$$
\left.\sup _{t \in J} \mathbf{E} \| \Phi x_{1}(t)-\Phi x_{2}(t)\right) \|_{X}^{2}
$$

$$
\begin{aligned}
& =\sup _{t \in J} \mathbf{E} \| \frac{1}{\Gamma(\alpha)} \int_{0}^{t}(t-s)^{\alpha-1} A(s)\left[x_{1}(s)-x_{2}(s)\right] \mathrm{d} s \\
& +\frac{1}{(\Gamma(\alpha))^{2}} \int_{0}^{t}(t-s)^{\alpha-1}\left[A_{1}(s)-A(s)\right] \\
& B(T-s)^{1-\alpha} \tilde{L}_{T}^{-1} \\
& {\left[\int_{0}^{T}(T-s)^{\alpha-1} A(s)\left[x_{2}(s)-x_{1}(s)\right] \mathrm{d} s\right.} \\
& +\int_{0}^{T}(T-s)^{\alpha-1}\left[f\left(s, x_{2}(s)\right)-f\left(s, x_{1}(s)\right)\right] \mathrm{d} s \\
& +\int_{0}^{T}(T-s)^{\alpha-1}\left[\sigma\left(s, x_{2}(s)\right)\right. \\
& \left.\left.-\sigma\left(s, x_{1}(s)\right)\right] \mathrm{d} W(s)\right] \mathrm{d} s \\
& +\frac{1}{\Gamma(\alpha)} \int_{0}^{t}(t-s)^{\alpha-1}\left[f\left(s, x_{1}(s)\right)-f\left(s, x_{2}(s)\right)\right] \mathrm{d} s \\
& +\frac{1}{\Gamma(\alpha)} \int_{0}^{t}(t-s)^{\alpha-1}\left[\sigma\left(s, x_{1}(s)\right)\right. \\
& \left.-\sigma\left(s, x_{2}(s)\right)\right] \mathrm{d} W(s) \|^{2} \\
& \leq \frac{4 T^{2 \alpha-1} \sup _{t \in J} \mathbf{E}\left\|x_{1}(t)-x_{2}(t)\right\|_{X}^{2}}{(2 \alpha-1)(\Gamma(\alpha))^{2}} \\
& \times\left(\sup _{t \in J} A(t)+T L_{1}+L_{2}\right) \\
& \times\left(1+6 K \sup _{t \in J}\left\|A_{1}(t)\right\|^{2}\|B\|^{2}\right) \\
& \leq \rho_{2} \sup _{t \in J} \mathbf{E}\left\|x_{1}(t)-x_{2}(t)\right\|_{X}^{2} .
\end{aligned}
$$

Using (H7), we conclude that $\Phi$ is a contraction mapping and hence there exists a unique fixed point $x \in \mathcal{H}_{2}$ for $\Phi$. Therefore the system (5) is completely controllable.

\section{Integrodifferential systems}

In this section we consider stochastic fractional integrodifferential systems of the form

$$
\begin{aligned}
{ }^{C} D^{\alpha} x(t)= & A_{1} x(t)+f t, x(t), \int_{0}^{t} g(t, s, x(s)) \mathrm{d} s \\
& +\sigma\left(t, x(t), \int_{0}^{t} h(t, s, x(s)) \mathrm{d} s\right) \frac{\mathrm{d} W(t)}{\mathrm{d} t}, \\
\tau x(t)= & B_{1} u(t), \\
x(0)= & x_{0} \in \mathcal{H}_{2}^{0},
\end{aligned}
$$

where $g, h: J \times J \times X \rightarrow X$ are continuous functions. Let us assume the following hypotheses alternative to (H5) and (H6).

(H5)' Let

$$
\rho_{3}=\frac{3 M_{2} T^{2 \alpha-1}}{2 \alpha-1}\left(T^{2} L_{1} L_{3}+T\left(L_{1}+L_{2} L_{4}\right)+L_{2}\right)
$$




$$
\times\left(1+4 M_{2} C K\left\|B_{1}\right\|^{2}\left(\left\|A_{1}\right\|^{2}+\|A\|^{2}\right)\right)
$$

be such that $0 \leq \rho_{3}<1$.

(H6)' There exist constants $L_{i}>0, i=1, \ldots, 4$, such that

$$
\begin{aligned}
& \left\|f\left(t, x_{1}, y_{1}\right)-f\left(t, x_{2}, y_{2}\right)\right\|_{X}^{2} \\
& \quad \leq L_{1}\left(\left\|x_{1}-x_{2}\right\|_{X}^{2}+\left\|y_{1}-y_{2}\right\|_{X}^{2}\right), \\
& \left\|\sigma\left(t, x_{1}, y_{1}\right)-\sigma\left(t, x_{2}, y_{2}\right)\right\|_{\mathbb{L}_{2}^{0}}^{2} \\
& \quad \leq L_{2}\left(\left\|x_{1}-x_{2}\right\|_{X}^{2}+\left\|y_{1}-y_{2}\right\|_{X}^{2}\right), \\
& \left\|g\left(t, x_{1}, y_{1}\right)-g\left(t, x_{2}, y_{2}\right)\right\|_{X}^{2} \\
& \quad \leq L_{3}\left(\left\|x_{1}-x_{2}\right\|_{X}^{2}+\left\|y_{1}-y_{2}\right\|_{X}^{2}\right), \\
& \left\|h\left(t, s, x_{1}\right)-h\left(t, s, x_{2}\right)\right\|_{X}^{2} \\
& \quad \leq L_{4}\left\|x_{1}-x_{2}\right\|_{X}^{2}
\end{aligned}
$$

for all $x_{1}, x_{2}, y_{1}, y_{2} \in X$.

The integral equation equivalent to $(9)$ is obtained as in Section 2, i.e.,

$$
\begin{aligned}
& x(t) \\
& =E_{\alpha}\left(A t^{\alpha}\right) x_{0}+\int_{0}^{t}(t-s)^{\alpha-1}\left[E_{\alpha, \alpha}\left(A(t-s)^{\alpha}\right) A_{1}\right. \\
& \left.\quad-A E_{\alpha, \alpha}\left(A(t-s)^{\alpha}\right)\right] B u(s) \mathrm{d} s \\
& \quad+\int_{0}^{t}(t-s)^{\alpha-1} E_{\alpha, \alpha}\left(A(t-s)^{\alpha}\right) \\
& \quad f\left(s, x(s), \int_{0}^{s} g(t, r, x(r)) \mathrm{d} r\right) \mathrm{d} s \\
& \quad+\int_{0}^{t}(t-s)^{\alpha-1} E_{\alpha, \alpha}\left(A(t-s)^{\alpha}\right) \\
& \\
& \sigma\left(s, x(s), \int_{0}^{s} h(t, r, x(r)) \mathrm{d} r\right) \mathrm{d} W(s) .
\end{aligned}
$$

We obtain sufficient conditions for the controllability of the system (9) in the following theorem, similarly as in Theorem 2 .

Theorem 4. If the hypotheses of Theorem 1 along with (H5)' and (H6)' are satisfied, then the boundary control nonlinear stochastic fractional system (9) is completely controllable.

To prove the above result, we define the operator $\Phi$ on $\mathcal{H}_{2}$ as

$$
\begin{aligned}
& \Phi x(t) \\
& =E_{\alpha}\left(A t^{\alpha}\right) x_{0}+\int_{0}^{t}(t-s)^{\alpha-1}\left[E_{\alpha, \alpha}\left(A(t-s)^{\alpha}\right) A_{1}\right. \\
& \left.\quad-A E_{\alpha, \alpha}\left(A(t-s)^{\alpha}\right)\right] B u(s) \mathrm{d} s \\
& \quad+\int_{0}^{t}(t-s)^{\alpha-1} E_{\alpha, \alpha}\left(A(t-s)^{\alpha}\right)
\end{aligned}
$$

$$
\begin{aligned}
& f\left(s, x(s), \int_{0}^{s} g(t, r, x(r)) \mathrm{d} r\right) \mathrm{d} s \\
& +\int_{0}^{t}(t-s)^{\alpha-1} E_{\alpha, \alpha}\left(A(t-s)^{\alpha}\right) \\
& \sigma\left(s, x(s), \int_{0}^{s} h(t, r, x(r)) \mathrm{d} r\right) \mathrm{d} W(s),
\end{aligned}
$$

where

$$
\begin{aligned}
u(t)= & (T-t)^{1-\alpha} \tilde{L}_{T}^{-1}\left(x_{1}-E_{\alpha}\left(A T^{\alpha}\right) x_{0}\right. \\
& -\int_{0}^{T}(T-s)^{\alpha-1} E_{\alpha, \alpha}\left(A(T-s)^{\alpha}\right) \\
& f\left(s, x(s), \int_{0}^{s} g(t, r, x(r)) \mathrm{d} r\right) \mathrm{d} s \\
& -\int_{0}^{T}(T-s)^{\alpha-1} E_{\alpha, \alpha}\left(A(T-s)^{\alpha}\right) \\
& \left.\sigma\left(s, x(s), \int_{0}^{s} h(t, r, x(r)) \mathrm{d} r\right) \mathrm{d} W(s)\right) .
\end{aligned}
$$

With the assumptions (H5)' and (H6)' we can prove that the operator $\Phi$ maps $\mathcal{H}_{2}$ into itself and

$$
\begin{aligned}
\left.\sup _{t \in J} \mathbf{E} \| \Phi x_{1}(t)-\Phi x_{2}(t)\right) \|_{X}^{2} & \\
& \leq \rho_{3} \sup _{t \in J} \mathbf{E}\left\|x_{1}(t)-x_{2}(t)\right\|_{X}^{2},
\end{aligned}
$$

which enables us to apply the Banach contraction principle to obtain complete controllability of the system (9) as we proceeded in Theorem 2

\section{Examples}

In this section we provide examples to illustrate the theory developed in the previous sections.

Example 1. Consider the stochastic fractional nonlinear system for $(t, x) \in[0, T] \times \mathbb{L}_{2}([0,1], \mathbb{R})=J \times X$,

$$
\begin{aligned}
{ }^{C} D^{\alpha} z(t, x)= & \int_{0}^{x} a(x-y) z(t, y) \mathrm{d} y \\
& +\frac{1}{30} \sin (z(t, x))+\frac{1}{30} z(t, x) \frac{\mathrm{d} W(t)}{\mathrm{d} t}, \\
z(t, x)= & u(t, x), \quad(t, x) \in J \times\{0,1\}, \\
z(0, x)= & z_{0}(x), \quad x \in[0,1] .
\end{aligned}
$$

Here $z: J \times[0,1] \rightarrow \mathbb{R}$ is a real valued function, ${ }^{C} D^{\alpha}$ is the Caputo derivative in time, $u \in \mathbb{L}_{2}([0, T] \times[0,1], \mathbb{R})$. The operator $A_{1}: \mathbb{L}_{2}([0,1], \mathbb{R}) \rightarrow \mathbb{L}_{2}([0,1], \mathbb{R})$ is defined as $A_{1} v(x)=\int_{0}^{x} a(x-y) v(y) \mathrm{d} y$, the operator $B_{1}$ : $\mathbb{L}_{2}([0,1], \mathbb{R}) \rightarrow \mathbb{R}^{2}=E$ is defined as $B_{1} u(t, x)=$ $(u(t, 0), u(t, 1))$, where $|u(t, 0)|$ or $|u(t, 1)|$ is greater than 
$\sup _{x \in[0,1]}|u(t, x)|$. Here $\tau: \mathbb{L}_{2}([0,1], \mathbb{R}) \rightarrow \mathbb{R}^{2}$ is defined by $\tau z(t, x)=(z(t, 0), z(t, 1))$. $W(t)$ is a real valued Wiener process. The operator $A$ is defined as

$$
\begin{array}{r}
D(A)=\{z \in X: z(0)=z(1)=0\}, \\
A z=A_{1} z \quad \text { for all } x \in D(A),
\end{array}
$$

and we see that $\|A\|^{2}=a^{2} / 6$. We have $D(A)=D(\tau)$ and we choose

$$
a<\frac{6 \sqrt{(2 \alpha-1) \Gamma(\alpha)}}{T^{\alpha}} .
$$

Then (H1) and (H2) are satisfied. We choose the bounded linear operator $B: \mathbb{L}_{2}([0,1], \mathrm{R}) \rightarrow \mathbb{L}_{2}([0,1], \mathbb{R})$ to be defined as $B u=w_{u}$, where $w_{u}$ is the solution of the following Volterra integral equation with two boundary conditions:

$$
\begin{aligned}
\int_{0}^{x} a(x-y) w_{u}(y) \mathrm{d} y & =w_{u}(x), & & x \in[0,1] \\
w_{u}(x) & =u(x), & & x \in\{0,1\} .
\end{aligned}
$$

Along with the assumption that either $u(0)$ or $u(1)$ is greater than $\sup _{x \in[0,1]} u(x)$, from the definition of the operator $B$, we get that the hypotheses (H3) and (H4) are satisfied from the following observations:

$$
\tau(B u)=(u(0), u(1))=B_{1} u
$$

and

$$
\begin{aligned}
E_{\alpha, \alpha}\left(A t^{\alpha}\right) B & \\
& =\sum_{k=0}^{\infty} \frac{t^{\alpha k} A^{k} B u(t)}{\Gamma(\alpha k+\alpha)} \in D(A), \quad t \in J,
\end{aligned}
$$

since from the definition of $A$ and $B$ we have, by 111, that $A B u(t, x)=B u(t, x)$, and from the boundary conditions on $w_{u}$ we have $A B u(t, x) \in D(A)$. The functions $f(t, z(t, x))=\sin (z(t, x))$ and $g(t, z(t, x))=$ $z(t, x)$ are Lipschitz continuous with Lipschitz constant $1 / 30$. Taking $T=1$, we see that the constant $\rho_{1}<1$ for different choices of $\alpha=0.4,0.6,0.7,0.8,0.9$. In particular for $\alpha=0.7$ we have $\rho_{1}=0.6740$. Thus all the hypotheses of Theorem 2 are satisfied and so the system (10) is completely controllable on the time interval $[0,1]$.

Example 2. Consider now the stochastic fractional time-varying nonlinear system for $(t, x) \in[0, T] \times$ $\mathbb{L}_{2}([0,1], \mathbb{R})=J \times X$

$$
\begin{aligned}
{ }^{C} D^{\alpha} z(t, x)= & a t \int_{0}^{x} z(t, y) \mathrm{d} y+\ln (\cosh (z(t, x))) \\
& +e^{-z(t, x)} \frac{\mathrm{d} W(t)}{\mathrm{d} t} \\
z(t, x)= & u(t, x), \quad(t, x) \in J \times\{0,1\}
\end{aligned}
$$

$$
z(0, x)=z_{0}(x), \quad x \in[0,1] .
$$

Here $z: J \times[0,1] \rightarrow \mathbb{R}$ is a real valued function, ${ }^{C} D^{\alpha}$ is the Caputo derivative in time, $u \in \mathbb{L}_{2}([0, T] \times[0,1], \mathbb{R})$.

The operator $A_{1}(t): \mathbb{L}_{2}([0,1], \mathbb{R}) \rightarrow \mathbb{L}_{2}([0,1], \mathbb{R})$ is defined as $A_{1}(t) v(x)=t \int_{0}^{x} a v(y) \mathrm{d} y$, the operator $B_{1}: \mathbb{L}_{2}([0,1], \mathbb{R}) \rightarrow \mathbb{R}^{2}=E$ is defined as $B_{1} u(t, x)=$ $(u(t, 0), u(t, 1))$, where $|u(t, 0)|$ or $|u(t, 1)|$ is greater than $\sup _{x \in[0,1]}|u(t, x)|$. Here $\tau: \mathbb{L}_{2}([0,1], \mathbb{R}) \rightarrow \mathbb{R}^{2}$ is defined by $\tau z(t, x)=(z(t, 0), z(t, 1)) . W(t)$ is a real valued Wiener process. The operators $A$ and $B$ can be constructed similarly as in to the previous example. The nonlinear functions $\ln (\cosh x)$ and $e^{-x}$ are Lipschitz continuous and $\rho_{2}$ can be made less than 1 by taking $T$ sufficiently small for a fixed $\alpha$. Thus all the hypotheses of Theorem 3 are satisfied and so the system (12) is completely controllable on the time interval $[0, \mathrm{~T}]$.

Example 3. Let $G=(0,1) \times(0,1)$ be an open subset of $\mathbb{R}^{2}$ whose boundary is denoted by $\partial G$. Set $S=\{(0,0),(0,1),(1,1),(1,0)\}$. Consider the nonlinear stochastic fractional system for $(t, x) \in[0, T] \times$ $\mathbb{L}_{2}(G, \mathbb{R})=J \times X$

$$
\begin{aligned}
{ }^{C} D^{\alpha} z(t, \mathbf{x})= & \frac{1}{\Gamma(\alpha)} \int_{0}^{\mathbf{x}} a(\mathbf{x}-\mathbf{y})^{\alpha-1} z(t, \mathbf{y}) \mathrm{d} \mathbf{y} \\
& +l_{1} \int_{0}^{t} \tan ^{-1}(z(s, \mathbf{x})) \mathrm{d} s \\
& +l_{2} z(t, \mathbf{x}) \frac{\mathrm{d} W(t)}{\mathrm{d} t}, \\
z(t, \mathbf{x})= & \begin{cases}u(t, \mathbf{x}), & (t, \mathbf{x}) \in J \times S, \\
0, & (t, \mathbf{x}) \in J \times \partial G \backslash S,\end{cases} \\
z(0, \mathbf{x})= & z_{0}(\mathbf{x}), \quad \mathbf{x} \in W .
\end{aligned}
$$

Here $\mathbf{x} \in \mathbb{R}^{2}, z: J \times G \rightarrow \mathbb{R}$ is a real valued function, ${ }^{C} D^{\alpha}$ is the Caputo derivative in time,

$$
u(t, \cdot) \in \mathbb{L}_{2}(G, \mathbb{R}) \in \mathbb{R}^{4}=U .
$$

The operator

$$
A_{1}: \mathbb{L}_{2}(G, \mathbb{R}) \rightarrow \mathbb{L}_{2}(G, \mathbb{R})
$$

is defined as

$$
A_{1} v(\mathbf{x})=\frac{1}{\Gamma(\alpha)} \int_{0}^{\mathbf{x}} a(\mathbf{x}-\mathbf{y})^{\alpha-1} v(\mathbf{y}) \mathrm{d} \mathbf{y} .
$$

Furthermore, $\tau: \mathbb{L}_{2}(G, \mathbb{R}) \rightarrow \mathbb{R}^{4}=E$ is defined by

$$
\begin{aligned}
& \tau z(t, x) \\
& \quad=(z(t,(0,0)), z(t,(0,1)), z(t,(1,1)), z(t,(1,0)))
\end{aligned}
$$

and

$$
B_{1}: \mathbb{L}_{2}(G, \mathbb{R}) \rightarrow \mathbb{R}^{4}
$$


is defined as

$$
B_{1} u(\mathbf{x})=(u(0,0), z(0,1), z(1,1), z(1,0)) .
$$

$W(t)$ is a real valued Wiener process. The operator $A$ is defined as

$$
\begin{aligned}
D(A)= & \left\{z \in \mathbb{L}_{2}(G, \mathbb{R}):\right. \\
& (z(0,0), z(0,1), z(1,1), z(1,0))=0\}, \\
A z= & A_{1} z, \quad \forall z \in D(A),
\end{aligned}
$$

and the constant $a$ can be chosen in such a way that (H2) is satisfied. We choose the bounded linear operator $B$ : $\mathbb{L}_{2}(G, \mathbb{R}) \rightarrow \mathbb{L}_{2}(G, \mathbb{R})$ so that $B u=w_{u}$, where $w_{u}$ is the solution of the following Volterra integral equation with four boundary conditions:

$$
\begin{aligned}
\frac{1}{6} \int_{0}^{\mathbf{x}}(\mathbf{x}-\mathbf{y})^{3} w_{u}(\mathbf{y}) \mathrm{d} \mathbf{y} & =w_{u}(\mathbf{x}), \quad \mathbf{x} \in G \\
w_{u}(x) & =u(x), \quad x \in S .
\end{aligned}
$$

The construction of $B$ is such that the hypotheses $(\mathrm{H} 3)$ and (H4) are satisfied. The functions $f(t, x, y))=l_{1} y$, $\sigma(t, x, y))=l_{2} x, g(t, s, x)=\int_{0}^{t} \tan ^{-1}(x(s)) \mathrm{d} s$ and $h(t, s, x)=0$ are all Lipschitz continuous and satisfy (H6)'. Again $T$ can be taken small enough such that $\rho_{3}<1$, and this proves that the system (13) is completely controllable by Theorem 4

\section{Conclusion}

Complete controllability of stochastic fractional systems with a bounded operator and control given on the boundary was obtained. Linear, semilinear and integrodifferential systems have been studied. The controllability results for the boundary control system have been obtained by constructing another system where the boundary control has been transformed to distributed control. Then the well-known method of solving the distributed control system has been used. The control was explicitly given in terms of a constructed pseudoinverse operator. Sufficient conditions for controllability of nonlinear systems have been obtained by using the Banach fixed point theorem. Examples have been constructed to illustrate the theory. Future goals could include obtaining controllability results for similar systems with unbounded operators.

\section{Acknowledgment}

The work of the first author is supported by the University Grant Commission under the grant no. MANF-2015-17-TAM-50645 from the Government of India. The second author is indebted to the University Grant Commission for providing a UGC-BSR Faculty Fellowship to carry out this work.

\section{References}

Balachandran, K. and Anandhi, E.R. (2001). Boundary controllability of integrodifferential systems in Banach spaces, Proceedings of the Indian Academy of Sciences (Mathematical Sciences) 111(1): 127-135, DOI: 10.1007/BF02829544.

Balachandran, K. and Divya, S. (2014). Controllability of nonlinear implicit fractional integrodifferential systems, International Journal of Applied Mathematics and Computer Science 24(4): 713-722, DOI: 10.2478/ amcs-2014-0052.

Balachandran, K. and Kokila, J. (2012). On the controllability of fractional dynamical systems, International Journal of Applied Mathematics and Computer Science 22(3): 523-531, DOI: 10.2478/v10006-012-0039-0.

Balachandran, K., Matar, M. and Trujillo, J.J. (2016). Note on controllability of linear fractional dynamical systems, Journal of Control and Decision 3(4): 267-279, DOI: 10.1080/23307706.2016.1217754.

Balakrishnan, A.V. (1977). Boundary control of parabolic equations, Theory of Nonlinear Operators-International Summer School, Berlin, Germany, pp. 113-124.

Barbu, V. (1980). Boundary control problems with convex cost criterion, SIAM Journal of Control and Optimization 18(1): 227-243. DOI: 10.1137/0318016.

Bashirov, A.E. (2003). Partially Observable Linear Systems under Dependent Noises, Springer, Boston, MA.

Curtain, R.F. and Zwart, H. (1995). An Introduction to Infinite Dimensional Systems Theory, Springer-Verlag, New York, NY.

Fattorini, H.O. (1968). Boundary control systems, SIAM Journal of Control and Optimization 6(3): 349-385, DOI: $10.1137 / 0306025$.

Gal, C. and Warma, M. (2016). Elliptic and parabolic equations with fractional diffusion and dynamic boundary conditions, Evolution Equations and Control Theory 5(1): 61-103, DOI: 10.3934/eect.2016.5.61.

Han, H.K. and Park, J.Y. (1999). Boundary controllability of differential equations with nonlocal condition, Journal of Mathematical Analysis and Application 230(1): 242-250, DOI: 10.1006/jmaa.1998.6199.

Hansen, S.W. (1994). Boundary control of a one-dimensional linear thermoelastic rod, SIAM Journal of Control and Optimization 32(4): 1052-1074, DOI: $10.1137 /$ S0363012991222607.

Kilbas, A., Srivastava, H.M. and Trujillo, J.J. (2006). Theory and Applications of Fractional Differential Equations, Elsevier, New York, NY.

Klamka, J. (1997). Constrained approximate boundary controllability, IEEE Transactions on Automatic Control 42(2): 280-284, DOI: 10.1109/9.554411.

Klamka, J. (2000). Constrained approximate controllability, IEEE Transactions on Automatic Control 45(9): 1745-1749. DOI: 10.1109/9.880640. 
Kreyszig, E. (1978). Introductory Functional Analysis with Applications, John Wiley and Sons, New York, NY.

Lagnese, J. (1977). Boundary value control of a class of hyperbolic equations in a general region, SIAM Journal of Control and Optimization 15(6): 973-983, DOI: $10.1137 / 0315062$.

Lasiecka, I. and Triggiani, R. (1991). Exact controllability of semilinear abstract systems with application to waves and plates boundary control problems, Applied Mathematics and Optimization 23(1): 109-154, DOI: 10.1007/ BF01442394.

Li, Y. and Liu, B. (2008). Boundary controllability of non-linear stochastic differential inclusions, Applicable Analysis 87(6): 709-722, DOI: 10.1080/ 00036810802213231.

Lions, J.L. and Magenes, E. (1972). Non-Homogeneous Boundary Value Problems and Applications, Springer-Verlag, New York, NY.

Mabel Lizzy, R., Balachandran, K. and Suvinthra, M. (2017). Controllability of nonlinear stochastic fractional systems with distributed delays in control, Journal of Control and Decision 4(3): 153-167, DOI: 10.1080/23307706.2017.1297690.

Mahmudov, N.I. (2001). Controllability of linear stochastic systems in Hilbert spaces, Journal of Mathematical Analysis and Applications 259(5): 64-82, DOI: $10.1109 / 9.920790$

Mahmudov, N.I. (2003). Controllability of semilinear stochastic systems in Hilbert spaces, Journal of Mathematical Analysis and Applications 288(1): 197-211.

Mainardi, F., Mura, A. and Pagnini, G. (2010). The M-Wright function in time-fractional diffusion processes: A tutorial survey, International Journal of Differential Equations 2010(104505): 1-29.

Oprzędkiewicz, K., Gawin, E. and Mitkowski, W. (2016). Modelling heat distribution with the use of a non-integer order, state space model, International Journal of Applied Mathematics and Computer Science 26(4): 749-756, DOI: 10.1515/amcs-2016-0052.

Picard, R., Trostorff, S. and Waurick, M. (2012). On a class of boundary control problems, Operators and Matrices 1(1): 185-204, DOI: 10.7153/oam-08-10.

Podlubny, I. (1999). Fractional Differential Equations, Academic Press, New York, NY.

Quinn, M.D. and Carmichael, N. (1985). An approach to nonlinear control problem using fixed point methods, degree theory, pseudo-inverse, Numerical Functional Analysis Optimization 7(2): 197-219, DOI: 10.1080/01630568508816189.
Triggiani, R. (1975). Controllability and observability in Banach space with bounded operators, SIAM Journal of Control and Optimization 13(2): 462-491.

Washburn, D. (1979). A bound on the boundary input map for parabolic equations with application to time optimal control, SIAM Journal of Control and Optimization 17(5): 652-671, DOI: 10.1137/0317046.

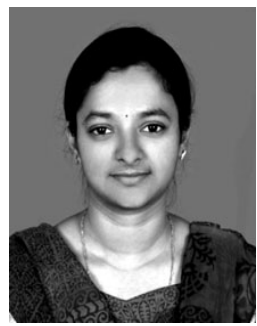

Rajendran Mabel Lizzy received her BSc and MSc degrees in mathematics from Bharathiar University in 2012 and 2014, respectively. She works there as a research scholar under the guidance of Prof. K. Balachandran in the Department of Mathematics. Her research interests include stochastic and fractional differential equations and control theory.

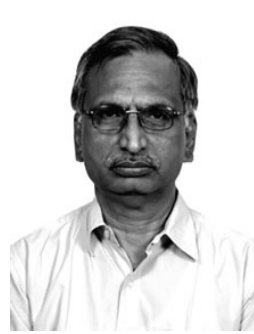

Krishnan Balachandran is a UGC BSR faculty fellow at the Department of Mathematics, Bharathiar University, Coimbatore, India. $\mathrm{He}$ received a Fulbright Award (1996), a Chandna Award (1999) and a Tamil Nadu Scientists Award (1999) for his excellent research contributions. His major research areas are control theory, abstract integrodifferential equations, stochastic differential equations, fractional differential equations and partial differential equations. He is a member of the AMS, IMS, CMS, ISIAM and ISCA. He is also a fellow of the Academy of Sciences, Chennai.

Received: 12 April 2017

Revised: 12 July 2017

Re-revised: 16 August 2017

Accepted: 1 September 2017 